\title{
Stochastic and Chaotic Relaxation Oscillations
}

\author{
J. Grasman ${ }^{1,2}$ and J. B. T. M. Roerdink ${ }^{1}$ \\ Received May 27, 1987; final September 27, 1988
}

\begin{abstract}
For relaxation oscillators stochastic and chaotic dynamics are investigated. The effect of random perturbations upon the period is computed. For an extended system with additional state variables chaotic behavior can be expected. As an example, the Van der Pol oscillator is changed into a third-order system admitting period doubling and chaos in a certain parameter range. The distinction between chaotic oscillation and oscillation with noise is explored. Return maps, power spectra, and Lyapunov exponents are analyzed for that purpose.
\end{abstract}

KEY WORDS: Relaxation oscillation; stochastic perturbation; chaos.

\section{INTRODUCTION}

Physical systems with periodic behavior usually show fluctuations in the period. One is inclined to ascribe this to perturbations coming from the environment (noise). The possibility of chaotic behavior of a deterministic system of three or more nonlinear differential equations has turned attention to a possible explanation in this direction.

In this paper we explore the distinction between stochastic and chaotic relaxation oscillations. A general theory for Van der Pol-type relaxation oscillators with stochastic perturbations is formulated in Section 3. We derive equations by which the probability distribution of the time between two jumps can be approximated. As an example, we study in detail the Van der Pol oscillator with a random forcing and compute the mean and variance of the interjump time for small noise intensity. In Section 4 it is

\footnotetext{
${ }^{1}$ Department of Applied Mathematics, Centre for Mathematics and Computer Science, Amsterdam, The Netherlands.

${ }^{2}$ Present address: Department of Mathematics, State University at Utrecht, The Netherlands.
} 
shown that a generalized Van der Pol-type oscillator may exhibit chaotic dynamics. As a prototype of such a system, we consider the Van der Pol oscillator with an additional state variable. In Section 5 this system is perturbed by noise and the dependence of the Lyapunov exponents upon the noise strength $\delta$ is investigated. Finally, in Section 6 we discuss various methods to detect the presence of a strange attractor in the noisy system from a time series of an output signal. We deal with power spectra, return maps, and Lyapunov exponents. Among these characteristics, the Lyapunov exponents yield the most definite information about the presence of a strange attractor.

The Van der Pol relaxation oscillator is the classical example of a nonlinear oscillator with the specific temporal structure as if it is periodically reset in its initial state. The equation reads

$$
\frac{d^{2} x}{d \tau^{2}}+v\left(x^{2}-1\right) \frac{d x}{d \tau}+x=0
$$

which is equivalent, using the transformations

$$
\tau=t v, \quad v=1 / \sqrt{\varepsilon}
$$

appropriate to the singularly perturbed case, to the system

$$
\begin{aligned}
\varepsilon \frac{d x}{d t} & =y-\frac{1}{3} x^{3}+x \\
\frac{d y}{d t} & =-x
\end{aligned}
$$

For $\varepsilon \rightarrow 0$ the limit cycle makes two jumps: from $A$ to $B$ and from $C$ to $D$ (see Fig. 1). At the arcs $B C$ and $D A$ the trajectory satisfies $y=\frac{1}{3} x^{3}-x$. Substitution in $(1.1 \mathrm{~b})$ yields an expression for the period:

$$
\frac{1}{2} T=\int_{2}^{1} \frac{x^{2}-1}{-x} d x=\frac{3}{2}-\ln 2
$$

In a more refined approach of small but nonzero values of $\varepsilon$, where the jumps are replaced by boundary layer approximations, one obtains

$$
T(\varepsilon) \approx 3-2 \ln 2+3 \alpha \varepsilon^{2 / 3}+O(\varepsilon \ln \varepsilon) \quad(\varepsilon \rightarrow 0)
$$

where $-\alpha=-2.33811$ is the first zero of the Airy function. ${ }^{(8)}$ Equation 


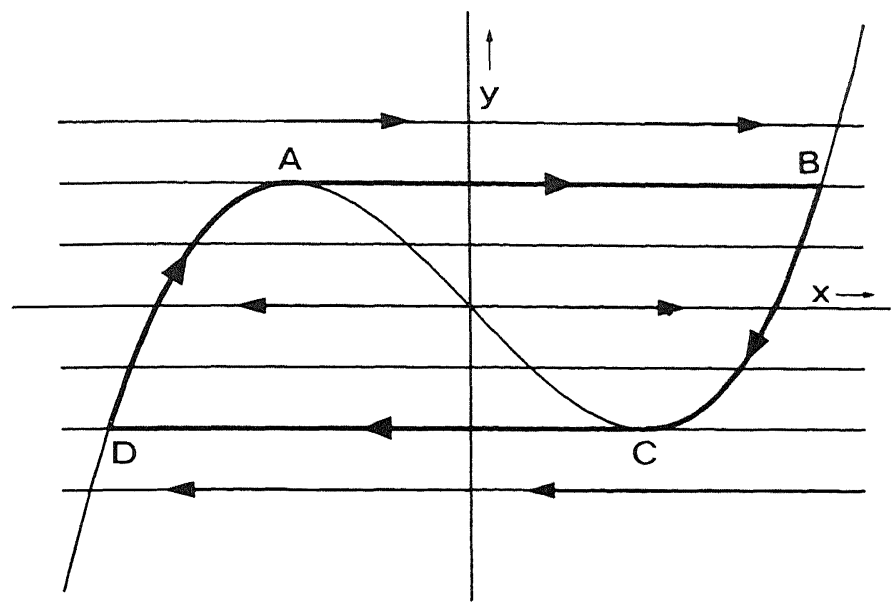

Fig. 1. Trajectories of the system (1.1) in the limit $\varepsilon \rightarrow 0$. For any starting value (the origin excluded) the solution approaches the limit cycle $A B C D$.

(1.1) can be viewed as a representative of a large class of nonlinear oscillators. It is therefore worthwhile to analyze the effect of small changes which turn this oscillator into a stochastic or chaotic system.

\section{THE GENERALIZED VAN DER POL OSCILLATOR}

The relaxation oscillations we consider are described by a system of differential equations of the form

$$
\begin{array}{rlrl}
\varepsilon \frac{d x_{i}}{d t} & =F_{i}(x, y ; \varepsilon), & i=1, \ldots, m \\
\frac{d y_{j}}{d t} & =G_{j}(x, y ; \varepsilon), \quad j=1, \ldots, n
\end{array}
$$

where $\varepsilon$ is a small, positive parameter. Here the $\left\{x_{i}\right\}$ represent the variables which undergo fast relaxation and the $\left\{y_{i}\right\}$ represent the slow variables. It is assumed that the system (2.1) has a relaxation oscillation (for the definition see ref. 8) as a solution.

If we let $\varepsilon \rightarrow 0$, the so-called discontinuous approximation of the oscillation is found which holds over a large phase of the cycle. The approximating trajectory satisfies

$$
\frac{d y}{d t}=G(x, y ; 0)
$$


and is restricted to the manifold

$$
\mathscr{F}=\{(x, y) \mid F(x, y ; 0)=0\}
$$

A trajectory of (2.1) remains only near $\mathscr{F}$ if, for $y$ fixed, the stationary solution $x=x_{s}$ of $(2.1 \mathrm{a})$ is stable with $x_{s}$ satisfying $F\left(x_{s}, y ; 0\right)=0$ and the point $\left(x_{s}, y\right)$ near the trajectory. When this is not the case, the trajectory will exhibit a large change in $x$ over a short time interval of length $O(\varepsilon)$. It is is assumed that indeed a subset $\mathscr{S}$ of $\mathscr{F}$ exists for which the matrix

$$
A=\left\{\frac{\partial F_{i}}{\partial x_{j}}\right\}_{m \times m}
$$

has eigenvalues with negative real parts: the stable manifold. At the boundary of $\mathscr{S}$ only one eigenvalue may have a real part in the form of a simple zero resulting in $\operatorname{det}(A)=0$. When the approximating trajectory arrives at a point $p \in \partial \mathscr{S}$, it leaves the manifold $\mathscr{F}$ and the solution jumps instantaneously to a point $r$ lying in $\mathscr{S}$ with $x_{r} \neq x_{p}$ and $y_{r}=y_{p}$. Clearly, the equation $F(x, y, 0)=0$ must be nonlinear in $x$.

As an example, we consider relaxation oscillations of a system with one "fast variable" $x$ and two "slow" variables $y_{1}$ and $y_{2}$. Expressing $x$ as a function of $y$, we obtain for $\mathscr{F}$

$$
x=H(y)
$$

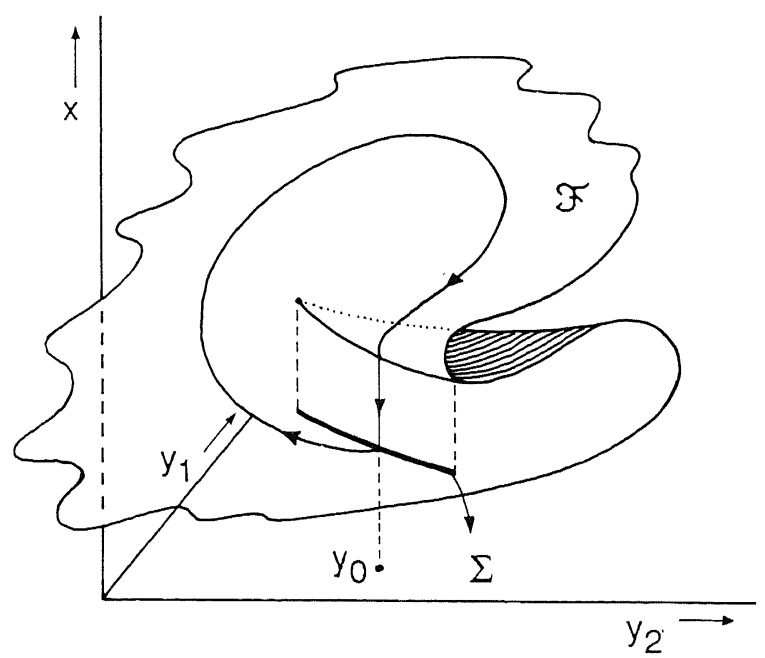

Fig. 2. Relaxation oscillation in a system with one fast and two slow variables. At $y=y_{0}$ the limit solution $(\varepsilon \rightarrow 0)$ jumps from one point of the manifold $\mathscr{F}$ to a different point, where (2.1a) with $(y, \varepsilon)=\left(y_{0}, 0\right)$ is stable. 
This expression is locally valid: at different branches of $\mathscr{F}$ different representations are needed. In Fig. 2 the manifold $\mathscr{F}$ is stable except for the middle branch at the fold. For any starting value away from $\mathscr{F}$ a trajectory of (2.1) will jump instantaneously (in the limit as $\varepsilon \rightarrow 0$ ) to one of the stable branches. The periodic solution has a jump at $y=y_{0}$. Its period satisfies

$$
T=\oint \frac{1}{G(H(y), y)} d s \quad(\varepsilon \rightarrow 0)
$$

where the integral is over the closed curve of the periodic solution. Let us take an $(m-1)$-dimensional transverse intersection $\Sigma \subset \mathscr{F}$ of $(2.2)-(2.3)$ with $\operatorname{det}(A) \neq 0$ for all $s \in \Sigma$. Approximating trajectories with jumps at $\partial \mathscr{S}$ generate a Poincaré mapping

$$
P: \quad \Sigma \rightarrow \Sigma
$$

This mapping or a finite repetition of it may have a fixed point that corresponds to a periodic solution. When this fixed point is stable, the existence of a periodic solution of $(2.1)$ with $\varepsilon>0$ can be proved. ${ }^{(18)}$ As we will see in Section 4, this mapping may also have chaotic solutions, corresponding to chaotic behavior of the system.

\section{RANDOMLY PERTURBED OSCILLATORS}

First we will model the influence of random perturbations upon the generalized Van der Pol oscillator (2.1). Near $\mathscr{S}$, points in $\mathbb{R}^{m+n}$ with equal values of $x$ will remain close to the trajectory starting in $\mathscr{F}$. Consequently, perturbations in the $x_{i}$ directions will not change the velocity in the direction along $\mathscr{F}$. Since we wish to study fluctuations in the period, we only take into account perturbations in the slow variables $\left\{y_{i}\right\}$. Thus, we analyze the system of stochastic differential equations

$$
\begin{aligned}
\varepsilon d X_{i} & =F_{i}(X, Y) d t, \quad i=1, \ldots, m \\
d Y_{j} & =G_{j}(X, Y) d t+\delta \sum_{k=1}^{p} \sigma_{j k}(X, Y) d W_{k}, \quad j=1, \ldots, n
\end{aligned}
$$

where $W_{1}, \ldots, W_{p}$ denote $p$ independent Wiener processes. It is assumed that $0<\varepsilon \ll \delta \ll 1$.

In our perturbation analysis we let $\varepsilon \rightarrow 0$ and consider a jumping periodic solution $L_{0}$ of (2.1) and its stochastic perturbation given by (3.1). We will apply methods for stochastic differential equations as described by Gardiner. ${ }^{(7)}$ 


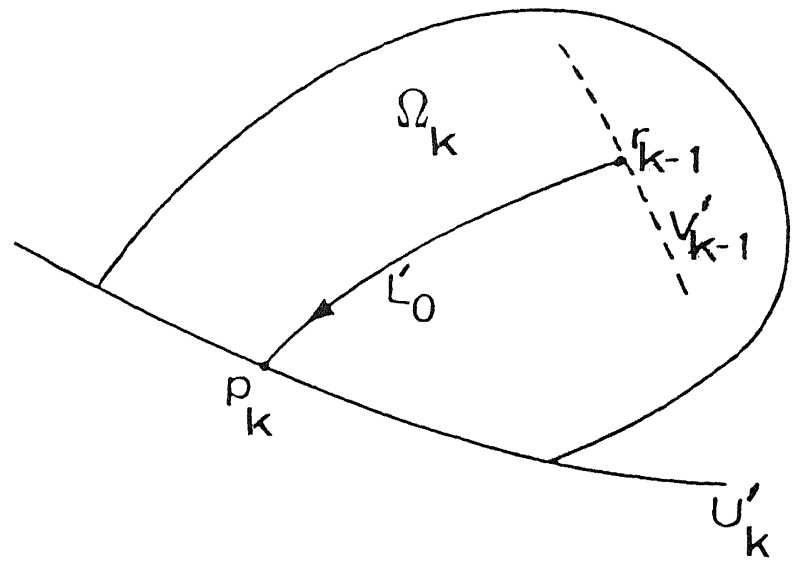

Fig. 3. A segment of the discontinuous limit cycle $L_{0}$ between a return point and a leaving point projected in $y$ space. In projection all trajectories leave $\Omega_{k}$ through $U_{k}^{\prime}$.

In the limit $\varepsilon \rightarrow 0$ we analyze the reduced system

$$
d y_{j}=G_{j}(H(y), y) d t+\delta \sum_{k=1}^{p} \sigma_{j k}(H(y), y) d W_{k}, \quad j=1, \ldots, n
$$

where $x=H(y)$ is a local solution of $F(x, y)=0$. The existence of a periodic solution $L_{0}$ for $\delta=0$ implies that the mapping $P$ of (2.7) has a stable fixed point. This closed trajectory contains a number of jumps, say $N$. The slow action is on $\mathscr{F}$ from a return point $r_{k}$ to a leaving point $p_{k+1}$. A jump is made from $p_{k}$ to $r_{k}, k=1, \ldots, N$. It is noted that $r_{N}$ is connected to $p_{1}$ by an interval of slow action. Let $U_{k} \subset \mathscr{F}$ be a set of points in a neighborhood of $p_{k}$ satisfying $\operatorname{det}(A)=0$, so $U_{k} \subset \partial \mathscr{S}$. Then a set of points $V_{k} \subset \mathscr{F}$ in a neighborhood of $r_{k}$ is defined by

$$
V_{k}=\left\{(x, y) \mid(x, y) \in \mathscr{S},(\bar{x}, y) \in U_{k} \text { for some } \bar{x}\right\}
$$

We will analyze stochastic trajectories in a domain $\Omega_{k}$ of the $y$ space that is a sufficiently large neighborhood of the projected segment $L_{0}^{\prime}$ of $L_{0}$, which connects the return point $r_{k-1}$ to the leaving point $p_{k}$ (see Fig. 3 ). ${ }^{3}$ The calculation of the distribution of the corresponding time interval proceeds by considering the part of the boundary $\partial \Omega_{k}$ formed by the set $U_{k}^{\prime}$ to be absorbing. The remaining part of $\partial \Omega_{k}$ is assumed to be at a sufficiently large distance of $L_{0}^{\prime}$ so that it does not play any role in the stochastic analysis.

\footnotetext{
${ }^{3}$ Here and in the following $S^{\prime}$ is the projection of a set $S$ onto the $y$ space for any $S \in \mathbb{R}^{m+n}$.
} 
We consider stochastic trajectories of (3.2) starting in $V_{k-1}^{\prime}$ up to the moment they reach the boundary at $U_{k}^{\prime}$. The time $T_{k}$ needed for this is a stochastic variable, called the interjump time. A method for approximating its distribution will be developed. For that purpose we first need to have information about the distribution of points $p_{k}$ at $U_{k}^{\prime}\left(=V_{k}^{\prime}\right)$ for a stationary oscillation as it goes through the $k$ th jump.

For a trajectory starting at $y \in \Omega_{k}$, let the probability of exit before time $t$ through a surface element $d S_{k}(u)$ located at the point $u \in U_{k}^{\prime}$ be denoted by

$$
g_{k}(u, y, t)\left|d S_{k}(u)\right|
$$

The probability density of ultimate exit through $d S_{k}(u)$ is then given by

$$
\pi_{k}(u, y)=g_{k}(u, y, \infty)
$$

and satisfies the Kolmogorov backward equation ${ }^{(7)}$

$$
\begin{gathered}
L_{\delta} \pi_{k} \equiv \sum_{i=1}^{n} G_{i}(y) \frac{\partial \pi}{\partial y_{i}}+\frac{1}{2} \delta^{2} \sum_{i, j=1}^{n} a_{i j}(y) \frac{\partial^{2} \pi}{\partial y_{i} \partial y_{j}}=0 \text { in } \Omega_{k} \\
\pi_{k}=\delta_{s}(u-y) \quad \text { for } y \text { on } \partial \Omega_{k} \text { and } u \in U_{k}^{\prime}
\end{gathered}
$$

where

$$
a_{i j}=\sum_{k=1}^{p} \sigma_{i k} \sigma_{k j}
$$

and with $\delta_{s}(u-y)$ a delta function defined on $\partial \Omega_{k}$ such that for any test function $f$,

$$
\int_{\partial \Omega_{k}} \delta_{s}(u-y) f(y)\left|d S_{k}(y)\right|=f(u), \quad u \in U_{k}^{\prime}
$$

If the distribution of starting points at $V_{k-1}^{\prime}$ for a stationary relaxation oscillation is denoted by $f_{k-1}(y)$, then

$$
f_{k}(u)=\int_{V_{k-1}^{\prime}} \pi(u, y) f_{k-1}(y)\left|d S_{k-1}(y)\right|
$$

is the distribution of arrival points at $U_{k}^{\prime}$ being also the stationary distribution of starting points at $V_{k}^{\prime}$. From the set of $N$ equations (3.9) with 
the index $k$ taken modulo $N$ we may determine $f_{k}(y), k=1,2, \ldots, N$. For small noise intensity $\delta$, the distribution is approximately of the form

$$
f_{k}(y)=\frac{C_{k}}{\delta^{n-1}} \exp \left\{-\frac{\left(y-y_{p}^{(k)}\right)^{T} A_{k}\left(y-y_{p}^{(k)}\right)}{\delta^{2}}\right\}
$$

and one obtains a set of relations among $A_{k}, k=1, \ldots, N$.

Next we consider the interjump time distribution. The time $T_{k}(y)$ needed to reach $U_{k}^{\prime}$ for the first time from a point $y$ belonging to $V_{k-1}^{\prime}$ is a random variable with density

$$
g_{k}(y, t)=\int_{U_{k}^{\prime}} g_{k}(u, y, t)\left|d S_{k}(u)\right|
$$

Its first and second moments $T_{k}^{(1)}(y)$ and $T_{k}^{(2)}(y)$ are defined by

$$
T_{k}^{(n)}(y)=\int t^{n} \frac{\partial}{\partial t} g_{k}(y, t) d t, \quad n=1,2
$$

They satisfy

$$
\begin{aligned}
L_{\delta} T_{k}^{(1)}=-1 & \text { in } \quad \Omega_{k} \\
T_{k}^{(1)}=0 & \text { on } \quad U_{k}^{\prime} \\
\partial T_{k}^{(1)} / \partial n=0 & \text { on } \quad \partial \Omega_{k} / U_{k}^{\prime}
\end{aligned}
$$

and

$$
\begin{aligned}
& L_{\delta} T_{k}^{(2)}=-2 T_{k}^{(1)} \\
& T_{k}^{(2)}=0 \quad \text { on } \quad U_{k}^{\prime} \\
& \partial T_{k}^{(2)} / \partial n=0 \quad \text { on } \quad \partial \Omega_{k} / U_{k}^{\prime}
\end{aligned}
$$

where the elliptic operator $L_{\delta}$ is defined by (3.6a). The unconditional probability density of the time $T_{k}$ between jumps $k-1$ and $k$ is

$$
g_{k}(t)=\int_{V_{k-1}^{\prime}} g_{k}(y, t) f_{k}(y)\left|d S_{k}(y)\right|
$$

with first and second moments given by

$$
T_{k}^{(n)}=\int_{V_{k-1}^{\prime}} T_{k}^{(n)}(y) f_{k}(y)\left|d S_{k}(y)\right|, \quad n=1,2
$$


Example. The Van der Pol oscillator with random forcing term. We consider the following stochastically perturbed Van der Pol oscillator:

$$
\begin{aligned}
\varepsilon d X & =\left(Y-\frac{1}{3} X^{3}+X\right) d t \\
d Y & =-X d t+\delta d W
\end{aligned}
$$

The local solutions of the equation

$$
y=\frac{1}{3} x^{3}-x
$$

are $x=H_{+}(y)$ for $x>1$ and $x=H_{-}(y)$ for $x<-1$ (see Fig. 1). In this case there are two jumps and, because of the symmetry, we only have to compute the distribution of one interjump time. The sets $\left\{U_{k}^{\prime}\right\}$ each contain just one point, viz. $y=2 / 3$ and $-2 / 3$, respectively.

Let us analyze the stochastic trajectories on the branch $x=H_{-}(y)$. The stochastic differential equation for the reduced problem $(\varepsilon=0)$ reads

$$
\begin{aligned}
d Y & =-H_{-}(Y) d t+\delta d W \\
Y(0) & =y, \quad y<2 / 3
\end{aligned}
$$

The domain $\Omega$ is bounded by a reflecting boundary at $y=-\infty$ and an absorbing one at $y=2 / 3$. For the problem (3.13) and (3.14) explicit solutions can be found ${ }^{(7)}$ :

$$
\begin{aligned}
& T^{(1)}(y ; \delta)=\frac{2}{\delta^{2}} \int_{y}^{2 / 3} \int_{-\infty}^{u} \exp \left\{\frac{2}{\delta^{2}}[R(u)-R(z)]\right\} d z d u \\
& T^{(2)}(y ; \delta)=\frac{2}{\delta^{2}} \int_{y}^{2 / 3} \int_{-\infty}^{u} T^{(1)}(z ; \delta) \exp \left\{\frac{2}{\delta^{2}}[R(u)-R(z)]\right\} d z d u
\end{aligned}
$$

where

$$
R(y)=\int_{-\infty}^{y} H_{-}(u) d u
$$

The integrals can be evaluated asymptotically for $0<\delta \ll 1$,

$$
T^{(n)}(y ; \delta)=T_{0}^{(n)}(y)+\delta^{2} T_{1}^{(n)}(y)+\cdots, \quad n=1,2
$$

We find, temporarily suppressing the $y$ dependences,

$$
\begin{aligned}
& T_{0}^{(1)}=-\frac{1}{2}+\frac{1}{2} H_{-}^{2}-\ln \left(-H_{-}\right) \\
& T_{0}^{(2)}=\left\{T_{0}^{(1)}\right\}^{2}
\end{aligned}
$$




$$
\begin{aligned}
& T_{1}^{(1)}=\frac{1}{4}\left(\frac{1}{H_{-}^{2}}-1\right) \\
& T_{1}^{(2)}=-\frac{1}{4}-\frac{1}{4} H_{-}^{2}+\frac{1}{2 H_{-}^{2}}+2 \ln \left(-H_{-}\right)
\end{aligned}
$$

Consequently, the interjump-time moments are found by substituting $y=-2 / 3$ and we have the following expected value and variance:

$$
\begin{aligned}
E(T) & =\left(\frac{3}{2}-\ln 2\right)-\frac{3}{16} \delta^{2}+O\left(\delta^{4}\right) \\
\operatorname{Var}(T) & =\left(-\frac{9}{16}+\frac{13}{8} \ln 2\right) \delta^{2}+O\left(\delta^{4}\right)
\end{aligned}
$$

To verify this result, a simulation of (3.17) was carried out by numerically solving the stochastic difference equations

$$
\begin{aligned}
& X(t+h)=X(t)+\frac{h}{\varepsilon}\left[Y(t)-\frac{1}{3} X^{3}(t)+X(t)\right] \\
& Y(t+h)=Y(t)-h X(t)+\delta h^{1 / 2} G(t)
\end{aligned}
$$

where $G(t)$ is a generator of random numbers with a normal distribution $N(0,1)$. For $\varepsilon=0.1$ and $\delta=0.25$ we find an agreement with (3.24) with an accuracy of 0.001 for a sample of about 100 interjump times. For $\delta$ sufficiently small a linear noise approximation can be made: the distribution of interjump times then has a normal distribution. For larger values of $\delta$, however, there will be nonnegligible contributions from the tail of the normal distribution with negative values of the interjump time, which are physically impossible. In that case it is proposed to use the inverse Gaussian distribution

$$
f(t ; \mu, \lambda)=\left(\frac{\lambda}{2 \pi t^{3}}\right)^{1 / 2} \exp \left[-\frac{\lambda(t-\mu)^{2}}{2 \mu^{2} t}\right], \quad t \geqslant 0
$$

as an approximation. Its expected value and variance are

$$
\begin{gathered}
E(T)=\mu \\
\operatorname{Var}(T)=\mu^{3} / \lambda
\end{gathered}
$$

If in (3.19a ) $H_{-}$were a positive constant, this density function would be 
exact. ${ }^{(11)}$ In Fig. 4 the distribution of 197 interjump times, numerically computed by $(3.25)$, is given in a histogram. For the values

$$
\varepsilon=0.1 \quad \text { and } \quad \delta=0.75
$$

the asymptotic theory yields

$$
\begin{aligned}
E(T) & =T_{0}(\varepsilon)-\frac{3}{16} \delta^{2}=\frac{3}{2}-\ln 2+\frac{3}{2} \alpha \varepsilon^{2 / 3}-\frac{3}{16} \delta^{2}=1.3 \\
\operatorname{Var}(T) & =\left(-\frac{9}{16}+\frac{13}{8} \ln 2\right) \delta^{2}=0.3
\end{aligned}
$$

Consequently, in the inverse Gaussian distribution function (3.26) we have to take

$$
\mu=1.3 \quad \text { and } \quad \lambda=7.4
$$

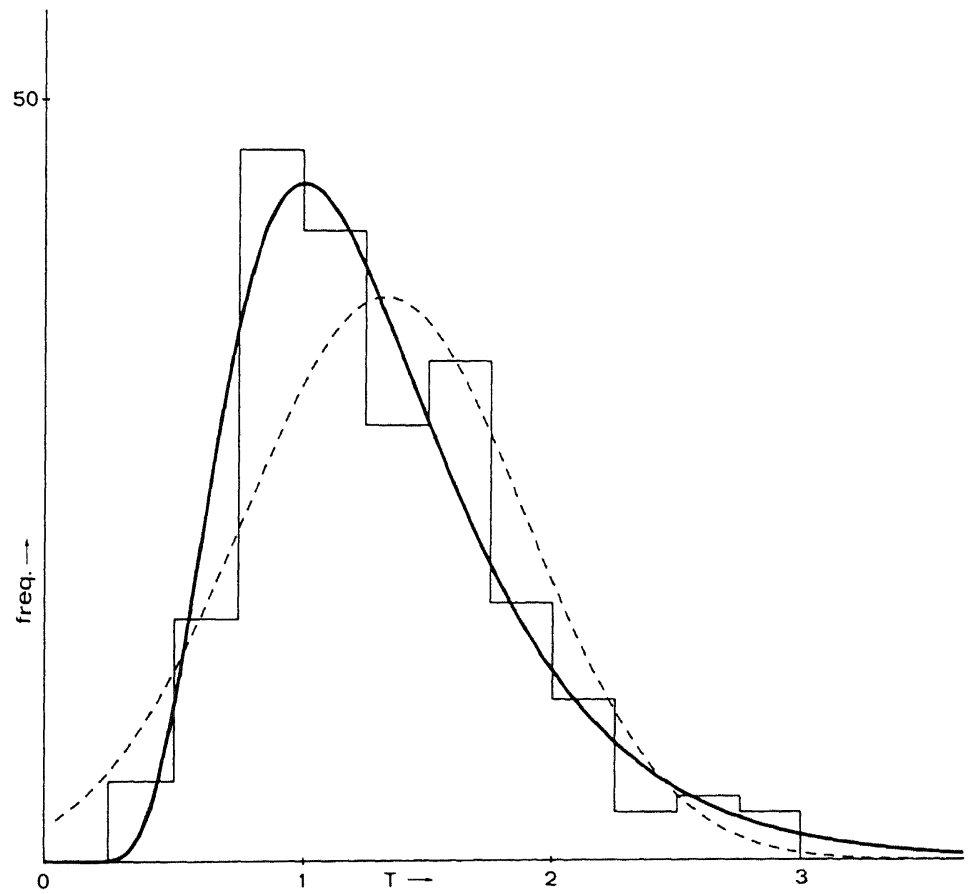

Fig. 4. Distribution of 197 interjump times from a simulation run of the system (3.17) and its approximation by a normal distribution (dotted line) and by an inverse Gaussian distribution (solid line). 
In Fig. 4 this distribution is represented by a solid line. It is noted that it fits the data much better than the normal distribution with the same mean and variance (dashed line).

\section{DETERMINISTIC CHAOTIC RELAXATION OSCILLATIONS}

From studies of Lorenz, ${ }^{(13)}$ Smale, ${ }^{(23)}$ Ruelle and Takens, ${ }^{(20)}$ and others it is known that continuous-time dynamical systems may exhibit chaotic behavior. It is quite possible to construct a system of type (2.1) possessing a limit solution that is a strange attractor and has some of the qualitative properties of a relaxation oscillation. By example we demonstrate that a system that remains most of the time in a two-dimensional manifold still may exhibit chaotic dynamics.

In Fig. 5 we sketch trajectories of a system with one fast and two slow variables. It is seen that for $\varepsilon \rightarrow 0$ the Poincare mapping of the compact interval $A B$ into itself agrees qualitatively with the logistic map. In this mapping the phenomena of period doubling and chaos are present. ${ }^{(2,17)}$

(a)

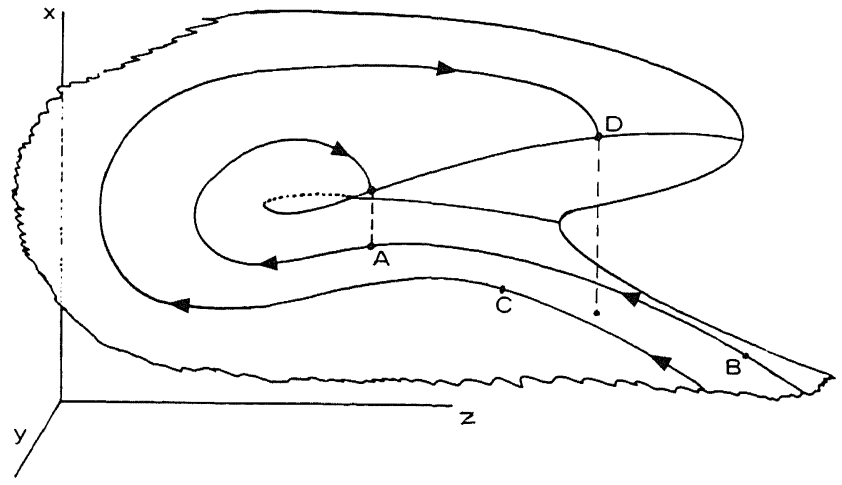

(b)

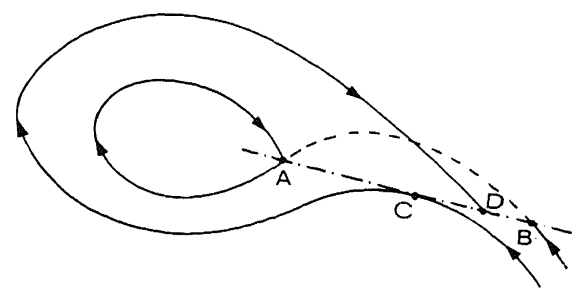

Fig. 5. A relaxation oscillator with chaotic dynamics. Following one cycle the trajectories generate a mapping of the curve $A B$ into itself. (a) Trajectories in state space, (b) projections in the $y, z$ plane. 
Examples of chaotic relaxation oscillations in fluid mechanics and biology are given by Lozi. ${ }^{(14)}$ Argémi and Rossetto ${ }^{(1)}$ model the irregular electrical activity of nerve cells by a chaotic relaxation oscillator. More systematic studies of chaotic dynamics in constrained equations $(\varepsilon=0)$ are presented by Ushiki and Lozi, ${ }^{(25)}$ Oka and Kokubu, ${ }^{(19)}$ and Takens. ${ }^{(24)}$

Example: A chaotic Van der Pol oscillator. The Van der Pol oscillator (1.1) can be augmented with a third state variable such that the resulting system of equations will have chaotic dynamics, while the regular Van der Pol relaxation oscillator is recovered if the third variable is set to zero. This is achieved by construction of a vector field similar to the one of Fig. 5. After some experimentation we found a system with the simplest polynomials

$$
\begin{aligned}
\varepsilon \frac{d x}{d t} & =y-\frac{1}{3} x^{3}+x \\
\frac{d y}{d t} & =-x-x^{2} z \\
\frac{d z}{d t} & =(x+a) z^{2}
\end{aligned}
$$

In Fig. 6a we sketch the trajectories in the $x, z$ plane for $\varepsilon \rightarrow 0$. The solution jumps from $x=1$ to $x=-2$ and from $x=-1$ to $x=2$. The Poincaré mapping $P_{0}$ of the line $(x, y, z)=(2,2 / 3, z)$ into itself is given in Fig. 6b. For $a<a_{1}$ one periodic solution is found; repeated period doubling occurs at $a_{k}$ with

$$
a_{1}=1.46, \quad a_{2}=1.59, \quad a_{3}=1.64, \ldots
$$

At $a_{\infty}=1.66$ the domain with chaotic solutions is entered.

For $a=1.7$ we computed the Lyapunov exponent of $P_{0}$, yielding $\lambda=0.31$. Taking $\varepsilon$ a fixed, positive constant, we may derive the Lyapunov exponents of a mapping $P_{\varepsilon}$ of a transverse plane into itself. Independent of the particular choice of the plane that is made, we obtain

$$
\lambda_{1}=0.51, \quad \lambda_{2}=-0.08 \quad \text { for } \quad \varepsilon=0.05
$$

Finally, using the scheme of Wolf et al., ${ }^{(26)}$ we compute the Lyapunov exponents of the flow of $(4.1)$ :

$$
\lambda_{1}=0.09, \quad \lambda_{2}=0, \quad \lambda_{3}=-26.45 \quad \text { for } \quad a=1.7, \quad \varepsilon=0.05
$$


(a)

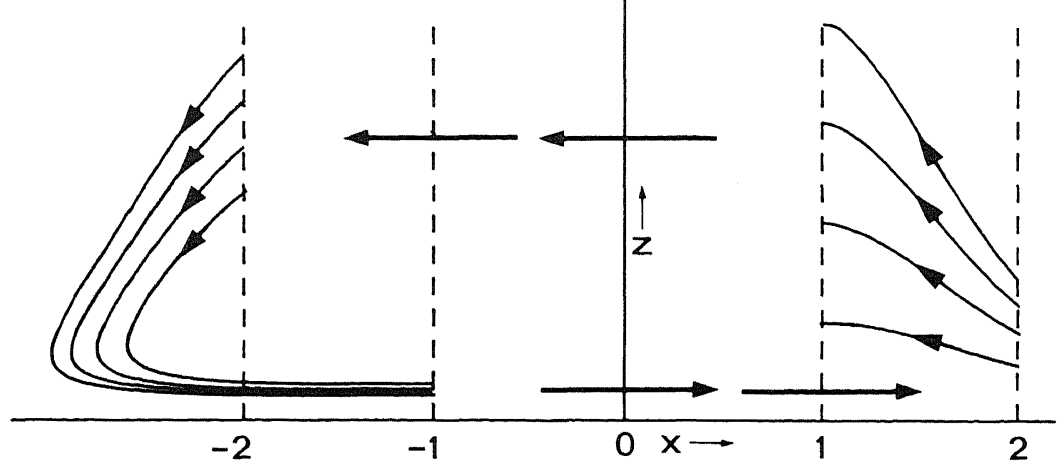

(b)

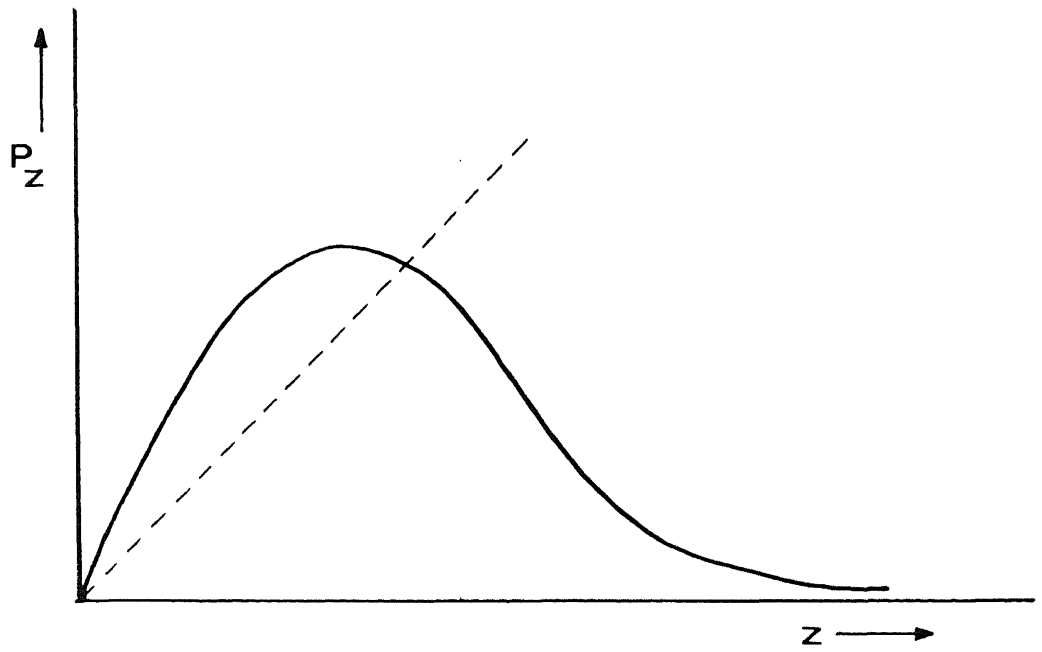

Fig. 6. A Van der Pol-type relaxation oscillator with a strange attractor in the limit $\varepsilon \rightarrow 0$.

(a) Trajectories projected in the $x, z$ plane; (b) sketch of the mapping at $(x, y)=(2,2 / 3)$.

The relation between the Lyapunov exponents $\lambda$ of the mapping $P_{0}$ and $\lambda_{1}$ of the flow (4.1) follows from their respective definitions:

$$
\lambda=\lim _{n \rightarrow \infty} \frac{1}{n} \sum_{k=0}^{n} \ln \left|P_{0}^{\prime}\left(z_{k}\right)\right|
$$

and

$$
\lambda_{1}(\varepsilon)=\lim _{t \rightarrow \infty} \frac{1}{t}{ }^{2} \log \frac{p(t)}{p(0)}
$$


where $p(t)$ is the length of the principal axis of the ellipsoid that evolves from a starting sphere at $t=0$. Consequently,

$$
\lim _{\varepsilon \rightarrow 0} \lambda_{1}(\varepsilon)=\lim _{n \rightarrow \infty}\left[T_{0}\left(z_{0}\right)+\cdots T_{0}\left(z_{n}\right)\right]^{-12} \log \left|\prod_{k=0}^{n} P_{0}^{\prime}\left(z_{k}\right)\right|
$$

or

$$
\lim _{\varepsilon \rightarrow 0} \lambda_{1}(\varepsilon)=\lambda /(\hat{T} \ln 2)
$$

Here $T_{0}\left(z_{k}\right)$ is the time between two successive crossings at $z=z_{k}$ and $z=z_{k+1}$ of the line $(x, y, z)=(2,2 / 3, z)$ for the system with $\varepsilon=0$, whereas $\hat{T}$ is the average interjump time, which is computed numerically from a long run in the same way as $\lambda$. We found $\hat{T}=5.04$, leading to $\lambda_{1}(0)=0.089$, which is close to the value (4.4) which holds for $\varepsilon=0.05$. A general discussion of the relation between $3 \mathrm{D}$ flows and $1 \mathrm{D}$ mappings is given by Shaw. ${ }^{(22)}$ For the converse of the procedure used here see Kottalam et al., ${ }^{(12)}$ who reduce the 3D chaotic Lorenz system to a 2D system perturbed by noise.

\section{CHAOTIC DYNAMICS PERTURBED BY NOISE}

The influence of external noise on a chaotic system has been extensively studied for $1 \mathrm{D}$ mappings. Crutchfield and Farmer $^{(3)}$ study the logistic mapping perturbed by noise. They find the disappearance of periodic windows and a lowered threshold for the transition to chaos. Moreover, the characteristic exponent $\lambda$ increases with the noise strength at parameter values corresponding to orbits which are periodic in the deterministic limit, whereas noise affects $\lambda$ very little at chaotic parameter values. On the other hand, Matsumoto and Tsuda find that noise may regularize chaotic dynamics, ${ }^{(15)}$ leading to a decrease of $\lambda$ with the noise strength $\delta$. They speak of noise-induced order. Effects of noise on ecological models are discussed by Schaffer et al. ${ }^{(21)}$

The effects of noise on many-dimensional flows is less well investigated. Zippelius and Lucke ${ }^{(27)}$ study the Lorenz model perturbed by external noise. For biochemical oscillators with chaotic orbits see Ebeling et al. ${ }^{(4)}$ and Herzel. ${ }^{(9)}$ The latter author finds that for large noise strength the maximum Lyapunov exponent may become negative: stabilization by noise. The forced Duffing oscillator with external noise is studied by Kapitaniak. ${ }^{(10)}$

Here we report results on the chaotic Van der Pol oscillator of the preceding section with a small white noise input:

$$
\varepsilon d X=\left(Y-1 / 3 X^{3}+X\right) d t
$$




$$
\begin{aligned}
& d Y=\left(-X-X^{2} Z\right) d t+\delta d W \\
& d Z=(X+a) Z^{2} d t
\end{aligned}
$$

where $W(t)$ is a Wiener process and $a=1.7$ corresponding to a chaotic orbit of the system (5.1) in the absence of noise.

To study the influence of the noise we have numerically calculated the Lyapunov exponents $\lambda_{1}, \lambda_{2}, \lambda_{3}$ along a stochastic trajectory of the system (5.1). Again we used the procedure of Wolf et al., ${ }^{(26)}$ but now augmented by a random generator for normally distributed jumps. A similar procedure was used by Crutchfield and Farmer ${ }^{(3)}$ for $1 \mathrm{D}$ maps with additive noise. The results are summarized in Table I. The value of $\varepsilon$ was fixed at $\varepsilon=0.05$ (decreasing $\varepsilon$ mainly affects $\lambda_{3}$ ). We observe that the first exponent $\lambda_{1}$ first slightly increases with $\delta$, after which a plateau value is reached. On the other hand, $\lambda_{2}$ decreases with $\delta$, whereas $\lambda_{3}$ is hardly affected. In contrast to the case of $1 \mathrm{D}$ maps where a single exponent describes the system, we now have three exponents whose response to increasing noise strength shows opposite tendencies. To obtain a measure of the average stability properties of the system (5.1), we follow Farmer et al. ${ }^{(6)}$ and compute the Lyapunov dimension $d_{L}$, which is defined as

$$
d_{L}=j+\sum_{i=1}^{j} \lambda_{i} /\left|\lambda_{j+1}\right|
$$

where $\lambda_{1} \geqslant \lambda_{2} \geqslant \cdots$ and $j$ is the largest integer such that $\lambda_{1}+\cdots \lambda_{j}>0$. The values of $d_{L}$ for various noise strengths are presented in the last column of Table I. Due to the limited numerical precision of the measurements, the quantitative values of $d_{L}$ are not very accurate. But it seems justified to draw the qualitative conclusion that a sudden decrease of $d_{L}$ occurs as $\delta$ increases. We interpret this as an indication that, although the maximum

Table I. Lyapunov Exponents $\left\{\lambda_{i}\right\}$ of the Flow (5.1) for Various Values of $\delta^{a}$

\begin{tabular}{lccrrr}
\hline$\delta$ & $\lambda_{1}$ & $\lambda_{2}$ & \multicolumn{1}{c}{$\lambda_{3}$} & \multicolumn{1}{c}{$\lambda$} & \multicolumn{1}{c}{$d_{L}$} \\
\hline 0 & $0.090 \pm 0.005$ & 0 & $-54 \pm 5$ & 0.31 & $2.00 \pm 0.30$ \\
0.1 & $0.105 \pm 0.005$ & $-0.055 \pm 0.005$ & $-56 \pm 5$ & 0.08 & $2.00 \pm 0.57$ \\
0.2 & $0.115 \pm 0.005$ & $-0.07 \pm 0.01$ & $-54 \pm 5$ & -0.06 & $2.00 \pm 0.85$ \\
0.3 & $0.12 \pm 0.01$ & $-0.16 \pm 0.02$ & $-53 \pm 5$ & -0.10 & $1.75 \pm 0.36$ \\
0.4 & $0.12 \pm 0.01$ & $-0.24 \pm 0.03$ & $-52 \pm 5$ & - & $1.50 \pm 0.25$ \\
0.5 & $0.12 \pm 0.01$ & $-0.26 \pm 0.03$ & $-45 \pm 5$ & - & $1.46 \pm 0.23$ \\
\hline
\end{tabular}

${ }^{a} \lambda$ is the exponent corresponding to the Poincare mapping $P_{0}^{(\delta)}(z) . d_{L}$ is the Lyapunov dimension (5.2). 
exponent $\lambda_{1}$ slightly increases with $\delta$, the system is stabilized in a global sense by the noise.

We may generalize the Poincare mapping $P_{0}$ defined in the previous section to a stochastic mapping $P_{0}^{(\delta)}(z)$ corresponding to oscillations perturbed by noise. In Fig. 7 we give the result using 25 values of $z$ with 16 simulation runs in each point. The noiseless curve $P_{0}:=P_{0}^{(0)}(z)$ is displayed for comparison. We also computed the Lyapunov exponent $\lambda$ of the mapping $P_{0}^{(\delta)}$ for a number of values of $\delta$. We took $P(z)=Q$, where $Q$ is a stochastic variable distributed according to a normal distribution $\mathscr{N}(m(z), s(z))$, where $m(z)$ and $s(z)$ are the mean and standard deviation of $P(z)$. For small $\delta$ the mapping can be approximated by

$$
z_{n}=m\left(z_{n-1}\right)+\delta N_{n}
$$

where $N_{n}$ is distributed according to the distribution $\mathcal{N}\left(0, s\left(z_{n-1}\right)\right)$, allowing us to use the derivative of $m(z)$ in the calculation of $\lambda^{(3)}$ The results are displayed in column 5 of Table I. Since there are various approximations involved in the derivation of the numerical algorithm for $\lambda$, these results have only a qualitative validity. The fact that $\lambda$ decreases toward negative values again indicates the stabilizing nature of the noise.

Finally, we have attempted to link the values of the exponents of the flow and the mapping, as we did in the noiseless case. To do this, one has to take into account the relative position of the evolving ellipsoid at the

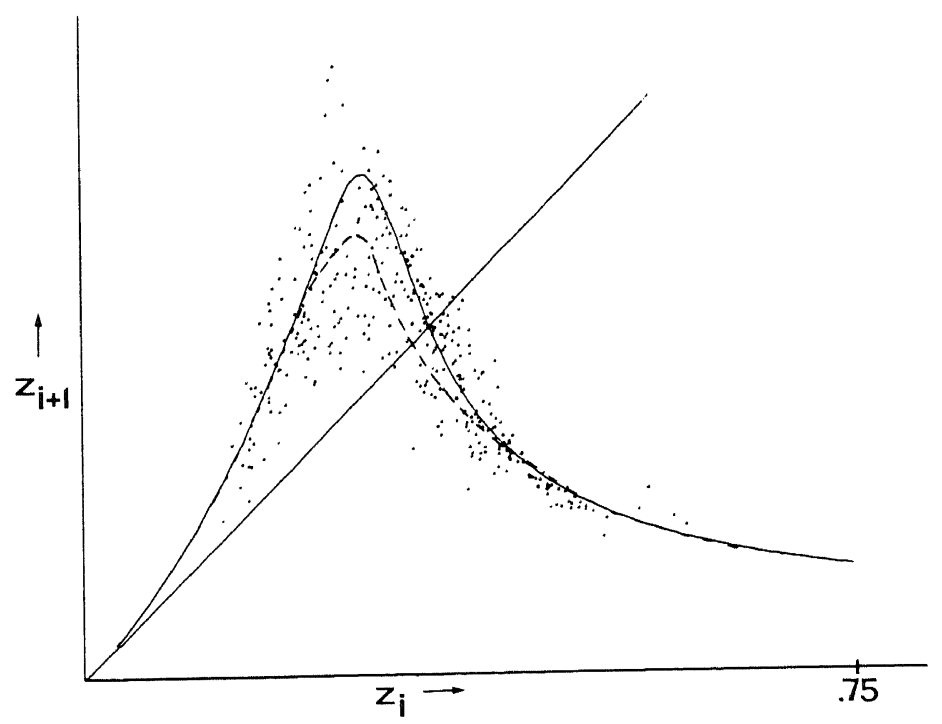

Fig. 7. Stochastic Poincaré mapping $P_{0}^{(\delta)}(z)$ for $\delta=0.1$. Solid line: the noiseless mapping $P_{0}$. Broken line: the average $m(z)$ for $\delta=0.1$. 
moment of the jump during the relaxation oscillation. For $\delta=0$ the long axis of the ellipsoid is perpendicular to the flow at the moment of the jump, but for increasing $\delta$ a systematic average rotation of the long axis with respect to this perpendicular direction was observed. However, we were unable to establish a quantitative relation between the exponents of the flow and mapping, respectively.

\section{ANALYSIS OF THE DYNAMICS FROM THE OUTPUT SIGNAL}

In the foregoing sections we analyzed oscillators with stochastic and/or chaotic dynamics from the explicitly given equations of motion. For

(a)

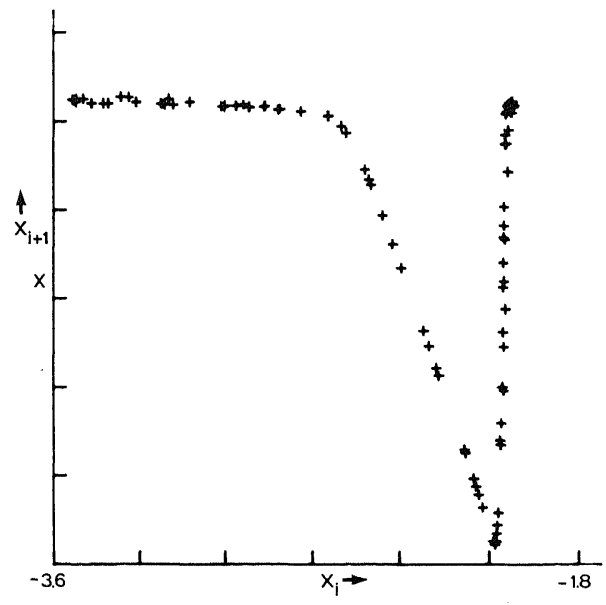

(b)

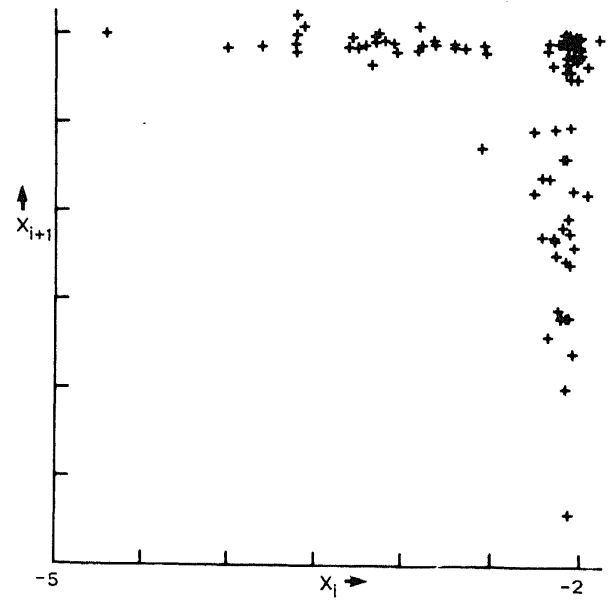

Fig. 8. Return map of the lowest value of $X$ in a cycle for the system (5.1). (a) $\delta=0$, (b) $\delta=0.3$. 
real physical systems the exact equations are not always known, so that one has to decide from an output signal about the internal dynamics of the system. When a physical system shows fluctuations in the period, one is inclined to ascribe this to perturbations coming from the environment (noise).

In a chemical system an intermediate reactant which is present for only a short time and therefore not noticed (hidden) may influence the dynamics and give rise to chaotic dynamics. The perturbation of the period has in that case a more fundamental cause. ${ }^{(4,5)}$ The question therefore arises whether one can distinguish chaotic from stochastic behavior without knowing the internal dynamics, even if the chaotic system is subjected to external noise.

(a)

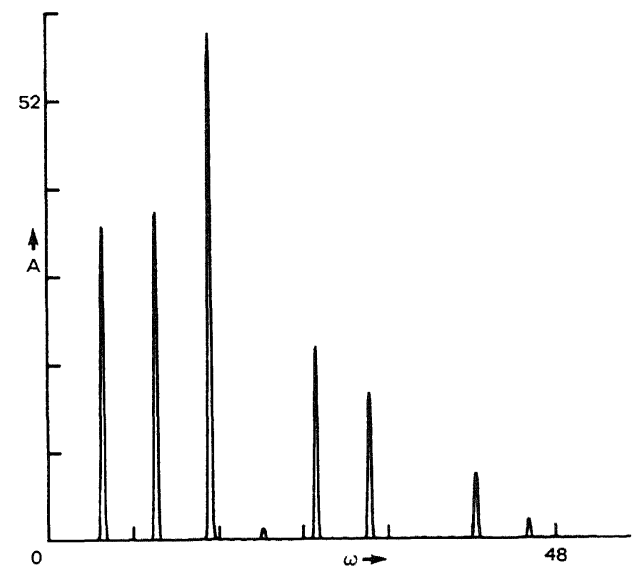

(b)

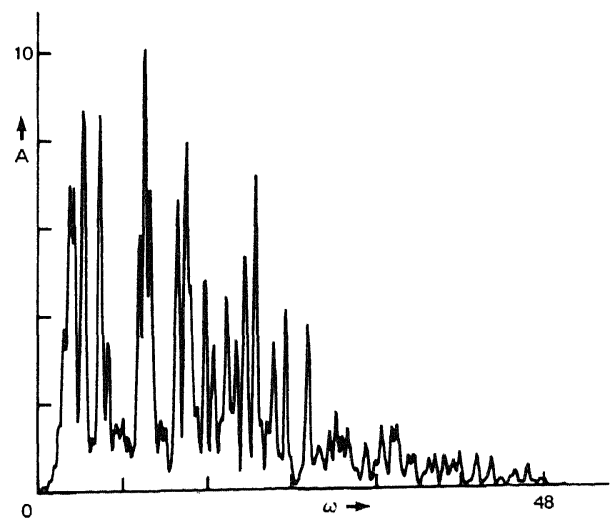

Fig. 9. Power spectra of the system (5.1). (a) $a=1.4, \delta=0$; (b) $a=1.4, \delta=0.3$. 
(c)

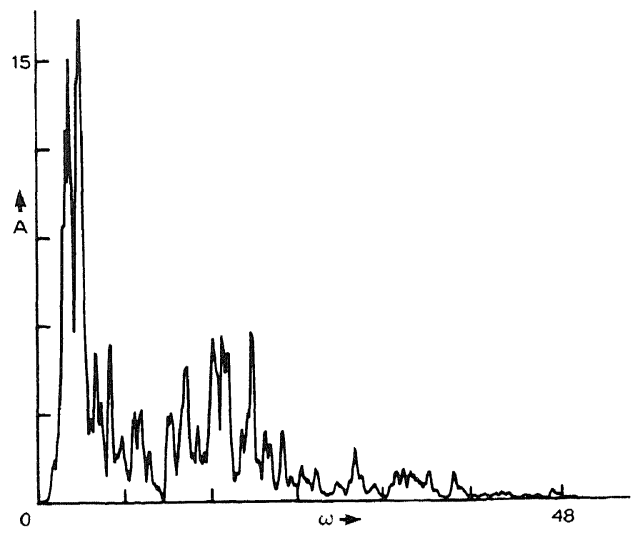

(d)

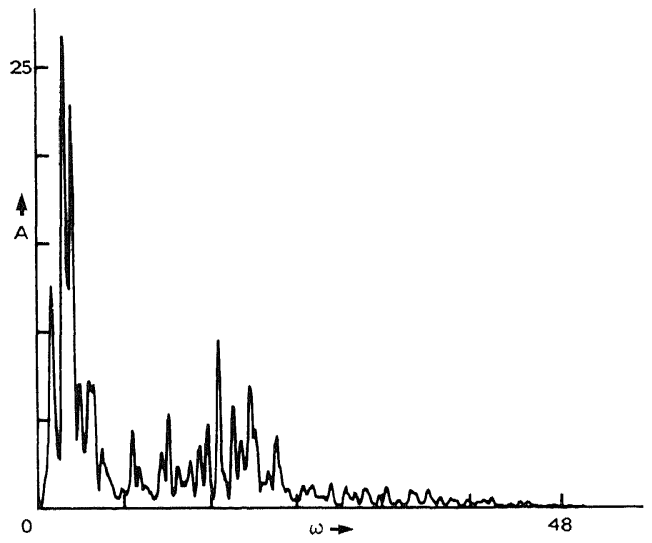

Fig. 9 (continued). (c) $a=1.7, \delta=0 ;$ (d) $a=1.7, \delta=0.3$.

We study this problem here by analyzing the dynamic properties of the system (5.1) from the output signal $X(t)$ only. For different values of $a$ and $\delta$ we take from the $x$ component a time series of 5000 points with a time step of 0.01 .

First we consider the return map for the lowest $x$ value over each cycle (see Fig. 8). For $a=1.7$ and $\delta=0$ the points form a Cantor set. For small noise a cloud of points around this set is expected. In Fig. $8 \mathrm{~b}$ we observe a breakdown of this cloud for $\delta=0.3$. From the clustering of data around a single point one would be inclined to conclude that the system is more or less periodic with some stochastic distribution. The power spectrum (see Fig. 9) confirms this observation: the peaks are higher and the area below 
(a)

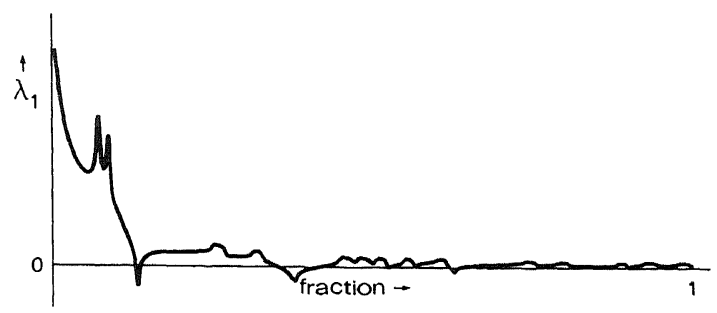

(b)

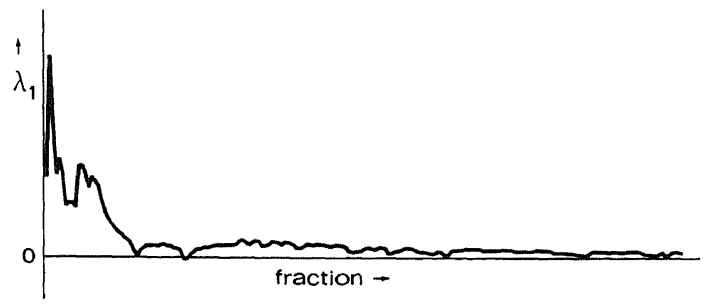

Fig. 10. Largest Lyapunov exponent from the data set as a function of the fraction of the time series that is used in the approximation process. (a) $a=1.7, \delta=0$; (b) $a=1.7, \delta=0.3$.

the curve has relatively decreased, which is in agreement with the analysis of Matsumoto. ${ }^{(16)}$

Finally, in Fig. 10 we plot the largest Lyapunov exponent $\lambda_{1}$ computed from the output signal as a function of the proportion of the time series that is used in the approximation process. ${ }^{(26)}$ The process converges to a value 0.075 with fluctuations of magnetude 0.025 , both in the case without $(\delta=0)$ and with noise $(\delta=0.3)$. Variation of the tuning parameters of the approximation process does not have any significant influence upon the value of the largest Lyapunov exponent. Clearly, the presence of chaotic dynamics can be detected quite well from the output signal by the method of Wolf et al. ${ }^{(26)}$ This criterion is more dependable than an inspection of the power spectrum or a return map, particularly when the signal is corrupted by noise. However, it is difficult to decide from the results based upon the output signal whether the addition of noise produces a change in $\lambda_{1}$. Such a change can be detected by computations based upon the explicit equations of motion, as we have shown in the previous section.

\section{REFERENCES}

1. J. Argémi and B. Rossetto, J. Math. Biol. 17:67-92 (1983).

2. P. Collet and J.-P. Eckmann, Iterated Maps on the Interval as Dynamical Systems (Birkhäuser, Basel, 1980). 
3. J. P. Crutchfield and J. D. Farmer, Phys. Rep. 92:45-82 (1982).

4. W. Ebeling, H. Herzel, and E. E. Sel'kov, in Proceedings 16th FEBS Congress, Part C (VNU Science Press, 1985), pp. 443-449.

5. H. Engel-Herbert, W. Ebeling, and H. Herzel, in Temporal Order, L. Rensing and N. I. Jaeger, eds. (Springer-Verlag, Berlin, 1985), pp. 144-152.

6. J. D. Farmer, E. Ott, and J. A. Yorke, Physica 7D:153-180 (1983).

7. C. W. Gardiner, Handbook of Stochastic Methods for Physics, Chemistry and the Natural Sciences (Springer-Verlag, Berlin, 1983).

8. J. Grasman, Asymptotic Methods for Relaxation Oscillations and Applications (SpringerVerlag, New York, 1987).

9. H. Herzel, Stabilization of Chaotic Orbits by Random Noise, Preprint, Humboldt University Berlin (1987).

10. T. Kapitaniak, Chaos in Systems with Noise (World Scientific, Singapore, 1988).

11. S. Karlin and H. M. Taylor, A First Course in Stochastic Processes (Academic Press, New York, 1975).

12. J. Kottalam, B. J. West, and K. Lindenberg, J. Stat. Phys. 46:119-133 (1987).

13. E. N. Lorenz, J. Atmos. Sci. 20:130-141 (1963).

14. R. Lozi, Modèles mathématiques qualitatifs simples et consistants pour l'étude de quelques systèmes dynamiques expérimentaux, Thesis, University of Nice (1983).

15. K. Matsumoto and I. Tsuda, J. Stat. Phys. 31:87-106 (1983).

16. K. Matsumoto, J. Stat. Phys. 34:111-127 (1984).

17. R. M. May, Nature 216:459-467 (1976).

18. E. F. Mishchenko and N. K. Rosov, Differential Equations with Small Parameters and Relaxation Oscillations (Plenum Press, New York, 1980).

19. H. Oka and H. Kokubu, Jpn. J. Appl. Math. 2:495-500 (1985).

20. D. Ruelle and F. Takens, Commun. Math. Phys. 20:167-192 (1971).

21. W. M. Schaffer, S. Ellner, and M. Kot, J. Math. Biol. 24:479-523 (1986).

22. R. Shaw, Z. Naturforsch. 36a:80-112 (1981).

23. S. Smale, Bull. Am. Soc. 73:747-817 (1967).

24. F. Takens, Transitions from periodic to strange attractors in constrained equations, Report ZW-8601, University of Groningen, The Netherlands (1986).

25. S. Ushiki and R. Lozi, Confinor and anti-confinor in constrained "Lorenz" system, preprint, University of Nice (1986).

26. A. Wolf, J. B. Swift, H. L. Swinney, and J. A. Vastano, Physica 16D:285-317 (1985).

27. A. Zippelius and M. Lücke, J. Stat. Phys. 24:345-358 (1981). 